\title{
Relative Humidity Facilitated Urea Particle Reaction with Salicyclic Acid: A Combined in situ Spectroscopy and DFT Study
}

Manoj Silva, ${ }^{1}$ Karolina Barcauskaite,,${ }^{1,2}$ Donata Drapanauskaite,,${ }^{1,2}$ Huijie Tian, ${ }^{1}$ Tomáš Bučko ${ }^{3}$ and Jonas Baltrusaitis ${ }^{1, *}$

1. Department of Chemical and Biomolecular Engineering, Lehigh University, B336 Iacocca Hall, 111 Research Drive, Bethlehem, Pennsylvania 18015, United States.

2.Lithuanian Research Centre for Agriculture and Forestry, Instituto al.1, LT-58344, Akademija, Kedainiai District, Lithuania.

3.Department of Physical and Theoretical Chemistry, Faculty of Natural Sciences, Comenius University in Bratislava, Ilkovičova 6, SK-84215, Bratislava, Slovak Republic

And Institute of Inorganic Chemistry, Slovak Academy of Sciences, Dúbravská cesta 9, SK-84236 Bratislava, Slovak Republic 
Table S1. Raman band assignments for urea and salicylic acid.

\begin{tabular}{|c|c|c|c|c|}
\hline \multicolumn{5}{|c|}{ Urea } \\
\hline $\begin{array}{l}\text { Raman spectrum } \\
\text { assignments mode }\end{array}$ & $\begin{array}{l}\text { Wavenumber } \\
\text { in the present }\end{array}$ & \multicolumn{2}{|c|}{$\begin{array}{l}\text { Wavenumber in other } \\
\text { experimental work }\left(\mathrm{cm}^{-1}\right)\end{array}$} & \multirow{2}{*}{$\left.\begin{array}{c}\begin{array}{c}\text { Wavenumber } \\
\text { in other }\end{array} \\
\text { computationa } \\
\text { work }\left(\mathbf{c m}^{-1}\right)\end{array}\right)$} \\
\hline C-N symmetric stretch & 1009 & & $1003^{1}$ & \\
\hline $\begin{array}{l}\text { N-C-N asymmetric } \\
\text { stretching }\end{array}$ & 1464 & $1468^{2}$ & $1466^{1}$ & $1473^{1}$ \\
\hline $\mathrm{NH}_{2}$ bending & 1536 & $1540^{2}$ & & \\
\hline C-O stretch & 1577 & $1578^{2}$ & $1540^{1}$ & $1554^{1}$ \\
\hline N-H stretch & 1645 & $1647^{2}$ & $1647^{1}$ & $1647^{1}$ \\
\hline \multicolumn{5}{|c|}{ Salicylic acid } \\
\hline $\mathrm{C}=\mathrm{O}$ stretch & 1632 & $1637^{3}$ & $1667^{4}$ & $1670^{3,4}$ \\
\hline $\begin{array}{l}\mathrm{C}-\mathrm{H} \text { phenolic ring in- } \\
\text { plane bend }\end{array}$ & 1028 & $1031^{3}$ & $1039^{4}$ & $1025^{4}$ \\
\hline $\begin{array}{l}\text { C-C phenolic ring } \\
\text { stretch }\end{array}$ & $\begin{array}{l}1469 \\
1583\end{array}$ & $\begin{array}{l}1474^{3} \\
1582^{3}\end{array}$ & $\begin{array}{l}1489^{4} \\
1596^{4}\end{array}$ & $\begin{array}{l}1474^{4} \\
1570^{4}\end{array}$ \\
\hline C-O stretch & $\begin{array}{l}1244 \\
1323\end{array}$ & $1248^{3}$ & $1248^{4}$ & $\begin{array}{l}1241^{4} \\
1320^{5}\end{array}$ \\
\hline $\mathrm{O}-\mathrm{H}$ in-plane bend & 1384 & $1362^{4}$ & & $1380^{4}$ \\
\hline $\begin{array}{l}\text { C-H in-plane } \\
\text { deformation }\end{array}$ & $\begin{array}{l}1149 \\
1166 \\
1090 \\
\end{array}$ & $\begin{array}{l}1148^{4} \\
1167^{4} \\
1093^{4}\end{array}$ & & $\begin{array}{l}1148^{4} \\
1190^{4} \\
1107^{4}\end{array}$ \\
\hline
\end{tabular}




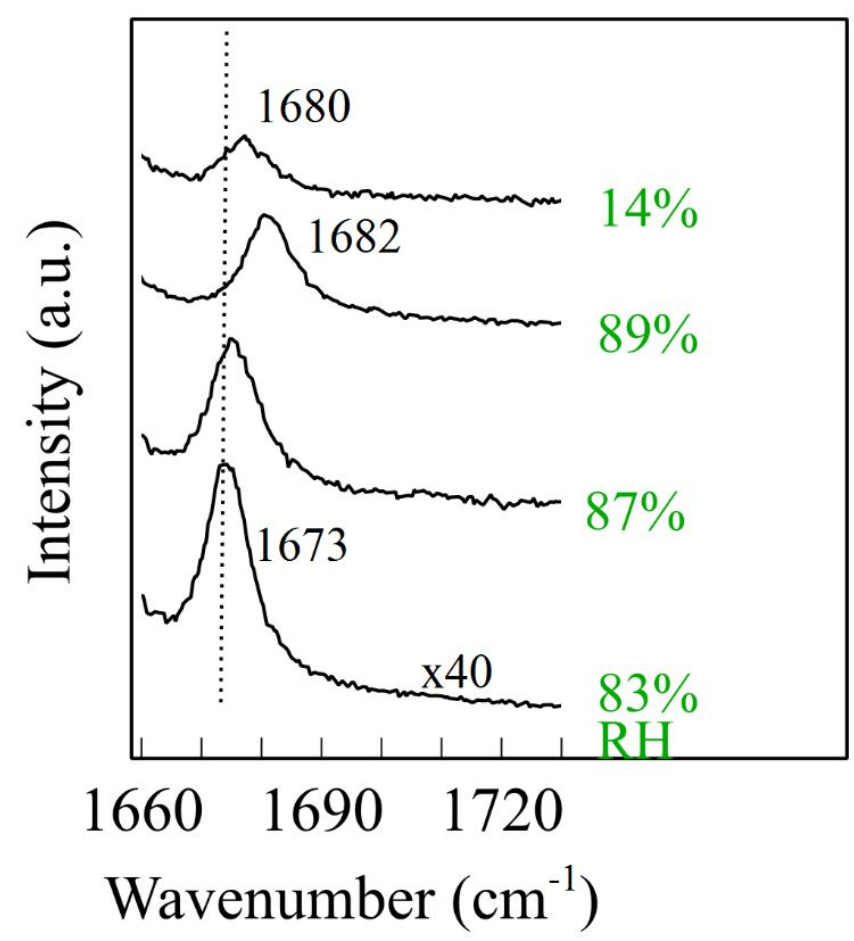

Figure S1. $1673 \mathrm{~cm}^{-1}$ evolution band showing the shift with increasing RH.

\section{Reference}

(1) Rousseau, B.; Keuleers, R.; Desseyn, H. O.; Geise, H. J.; Van Alsenoy, C. Solids Modeled by Ab-Initio Crystal Field Methods. Effects of Intermolecular Interactions on the Vibrational Spectrum of Urea. Chem. Phys. Lett. 1999, 302 (1-2), 55-59. https://doi.org/10.1016/S0009-2614(99)00108-6.

(2) Acosta-Maeda, T. E.; Misra, A. K.; Muzangwa, L. G.; Berlanga, G.; Muchow, D.; Porter, J.; Sharma, S. K. Remote Raman Measurements of Minerals, Organics, and Inorganics at 430 m Range. Appl. Opt. 2016, 55 (36), 10283. https://doi.org/10.1364/ao.55.010283.

(3) Volovšek, V.; Colombo, L.; Furić, K. Vibrational Spectrum and Normal Coordinate Calculations of the Salicylic Acid Molecule. J. Raman Spectrosc. 1983, 14 (5), 347-352. https://doi.org/10.1002/jrs.1250140511.

(4) Trout, C. C.; Tambach, T. J.; Kubicki, J. D. Correlation of Observed and Model Vibrational Frequencies for Aqueous Organic Acids: UV Resonance Raman Spectra and Molecular Orbital Calculations of Benzoic, Salicylic, and Phthalic Acids. Spectrochim. Acta - Part A Mol. Biomol. Spectrosc. 2005, 61 (11-12), 2622-2633. https://doi.org/10.1016/j.saa.2004.10.015.

(5) Socrates, G. Infrared and Raman Characteristic Group Frequencies. In Journal of Raman Spectroscopy; 2001; p 347. https://doi.org/10.1002/jrs.1238. 